\title{
Myocardial Protection through Pre- and Post-Conditioning: A Review of Mechanisms, Clinical Trials and Future Directions
}

Qamar Iqbal ${ }^{1}$ and Santiago Garcia ${ }^{2,3 *}$

${ }^{1}$ Department of Medicine, HealthEast Care System, St. Paul, MN, USA

${ }^{2}$ Department of Medicine, Minneapolis VA Healthcare System, Minneapolis, MN, USA

${ }^{3}$ Department of Medicine, University of Minnesota Medical School, Minneapolis, MN, USA

\begin{abstract}
Although the benefits of pre- and post-conditioning have been extensively documented in animal models, the translation from "bench to bedside" has been disappointing and slow. In this article we review the mechanisms of action and potential pharmacological targets of pre and post-conditioning, discuss key findings of clinical trials and provide a summary of ongoing clinical trials. Myocardial protection before planned myocardial ischemia and during reperfusion has the potential to significantly impact clinical outcomes. Industry and government support are critical to validate the findings of small-scale studies to the broader population with cardiovascular disease.
\end{abstract}

Keywords: Acute Myocardial Infarction; Clinical trials; Congestive Heart Failure; Percutaneous Coronary Intervention

\section{Introduction}

Morbidity and mortality from chronic coronary artery disease and Acute Myocardial Infarction (AMI) represents a significant public health burden in the United States and is the leading cause of death throughout the world [1]. Despite advances on multiple fronts to reduce ischemic injury from AMI such as thrombolytic therapy, primary Percutaneous Coronary Intervention (PCI) and establishment of regional networks for AMI transfer and care, many patients will develop post-AMI Left Ventricular (LV) dysfunction and Congestive Heart Failure (CHF), which is the leading hospital admission diagnosis in this country [2-6]. Additionally, many patients will also require placement of cardiac defibrillators and bi-ventricular pacemakers highlighting the critical need for identification and implementation of novel forms of cardioprotection in the setting of AMI [7-9].

Although the rapid restoration of coronary blood flow is the most effective means of reducing infarct size and preserving left-ventricular (LV) function, reperfusion may also be associated with further injury to the myocardium and vasculature [10]. Reperfusion injury may increase infarct size to a degree that is similar to the initial ischemic insult by increasing myocyte cell death, activation of apoptosis and promotion of endothelial dysfunction [11-13] (Figure 1). Unfortunately, reducing reperfusion injury replicating methods previously used in animal models has largely been unsuccessful in clinical trials [9]. To date, clinical trials to limit reperfusion injury have targeted many areas including Reactive Oxygen Species (ROS), reductions in calcium overload and $\mathrm{Na}^{+}-\mathrm{H}^{+}$ exchange inhibitors and the inflammation. Reasons for disappointing results in the majority of trials may include the much longer duration of ischemia in humans with AMI compared to animals and failure to deliver the therapy at the immediate onset of reperfusion. This point is essential since even a delay of several minutes following reperfusion may render these therapies ineffective [14]. The objective of this review is to summarize current knowledge on myocardial protection through pre- and post-conditioning with emphasis on mechanisms of action and clinical trials.

\section{Historical Notes on Myocardial Pre-conditioning}

Murry and coauthors were the first to demonstrate the concept of myocardial protection through pre-conditioning (PreC) [15]. The authors observed that repetitive episodes of ischemia, applied prior to a complete 40-minute duration circumflex artery occlusion, could beget protection $(75 \%$ reduction in infarct size was reported in this first experiment) instead of the logically anticipated myocardial damage. These authors were also the first to notice the time-dependence of PreC.

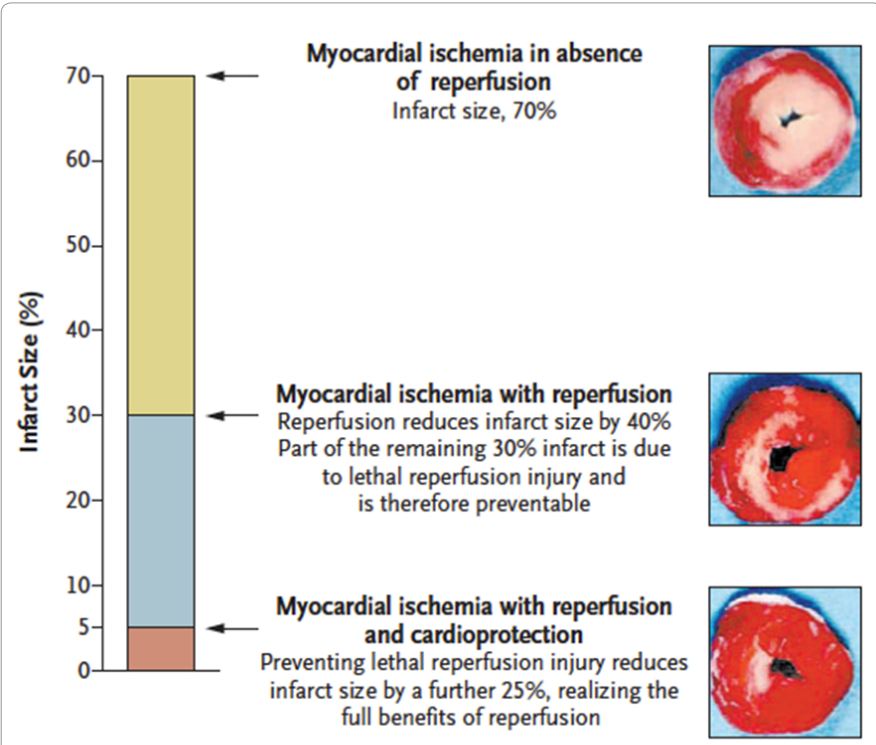

Figure 1: Quantification of reperfusion and ischemic injury in relationship to final infarct size in acute myocardial infarction. Reproduced from $\mathrm{N}$ Engl J Med 2007; 357: 1121-1135 with permission of Massachusetts medical Society.

${ }^{*}$ Corresponding author: Santiago Garcia, Assistant Professor of Medicine, One Veterans Drive (111-C), Minneapolis, MN 55417, USA, Tel: 612-467-3670; Fax 612-727-5668; E-mail: garci205@umn.edu

Received June 29, 2013; Accepted July 20, 2013; Published July 27, 2013

Citation: Iqbal Q, Garcia S (2013) Myocardial Protection through Pre- and PostConditioning: A Review of Mechanisms, Clinical Trials and Future Directions. J Cardiovasc Dis Diagn 1: 115. doi:10.4172/2329-9517.1000115

Copyright: (c) 2013 lqbal Q, et al. This is an open-access article distributed under the terms of the Creative Commons Attribution License, which permits unrestricted use, distribution, and reproduction in any medium, provided the original author and source are credited. 
In a second set of animals exposed to more prolonged ischemic times (3 hours) PreC was not associated with a reduction in infarct size [15].

Postconditioning (PosC), on the other hand, refers to episodes of ischemia -reperfusion applied after a complete arterial occlusion. A critical early observation was that reperfusion injury could be modified by slowly initiating reflow [16]. This "gentle" reperfusion resulted in smaller infarcts, reduced edema in the area at risk, and was associated with less "no reflow". Zhao et al. showed in an animal model that the benefits of PreC and PosC are of the same magnitude [16]. This is important, as most ischemic insults in clinical practice are not planned and therefore not amenable to PreC.

The concept of "Remote" Ischemic Preconditioning (RIPC) was first described by Przylenk et al. in 1993 [17]. The notion that repetitive episodes of ischemic-reperfusion in one circulatory bed would result in protection of other circulatory beds when exposed to an acute ischemic insult was first tested in the coronary circulation. Using anesthetized dogs, the authors found that 4 episodes of 5 minutes of circumflex coronary artery occlusion interspersed with 5 minutes of reperfusion before a 1-hour sustained Left Anterior Descending (LAD) coronary artery occlusion plus 4.5 hours of reperfusion reduced infarct size in the LAD bed to $6 \%$ of the area at risk (AAR) compared to $16 \%$ in controls. The magnitude of the benefit with RIPC applied to the Circumflex artery (35\%) was similar to that observed when PreC was applied directly to the LAD. The finding that PreC "at a distance" is as effective as Pre $\mathrm{C}$ allowed testing the concept of RIPC in several human clinical trials.

\section{Evaluation of Pre-Conditioning in Humans}

Several trials have assessed the benefits of PreC and RIPC in patients undergoing procedures involving "planned" myocardial ischemia such as coronary angioplasty or Coronary Artery Bypass Surgery (CABG) [18-21]. The most common circulatory bed for applying remote PreC has been the forearm by externally compressing the brachial artery with a blood pressure cuff. This is typically performed with a standard blood pressure cuff inflated over the patient's systolic blood pressure (usually to $200 \mathrm{mmHg}$ ) for five minutes and then deflated for 5 minutes to allow for reperfusion. This cycle is then repeated 3-5 times. The approach of inducing PreC by inflating a blood pressure cuff is noninvasive, safe, and technically feasible $[18,19]$.

Hoole et al. showed in 242 patients undergoing PCI that transient limb ischemia applied before arrival in the catheterization laboratory resulted in decreased median troponin I concentrations at 24 hours $(0.06 \mathrm{ng} / \mathrm{ml}$ vs. $0.16 \mathrm{ng} / \mathrm{ml}, \mathrm{P}=0.04)$ and numerically lower number of major adverse cardiac events at 6 months ( 4 vs. 13, $\mathrm{p}=0.018)$ [18].

Hausenloy et al. randomized 57 patients undergoing Coronary Artery Bypass Surgery (CABG) to RIPC or control. RIPC consisted of three 5-minutes cycles of right upper limb ischemia (cuff inflated to 200 $\mathrm{mmHg}$ ) and 5 minutes of reperfusion (cuff deflation) after induction of anesthesia. The total area under the curve for RIPC patients decreased by $43 \%(21 \mu \mathrm{g} / \mathrm{L}$ vs. $36 \mu \mathrm{g} / \mathrm{L}, \mathrm{p}=0.005)$ [19].

Ali et al. randomized 82 patients undergoing open Abdominal Aortic Aneurysm (AAA) repair to RIPC, induced by intermittent (10minutes cycles) cross clamping of the common iliac artery, or conventional surgery. RIPC was associated with a $27 \%$ reduction in the incidence of myocardial injury, assessed by troponin I level $>0.4 \mathrm{ng} / \mathrm{ml}$, a $22 \%$ reduction in the incidence of myocardial infarction, and a $23 \%$ reduction in the incidence of renal failure [20].
The beneficial effects of PreC are not restricted to patients with atherosclerosis-related ischemia. Cheung et al. randomized 37 pediatric patients undergoing corrective cardiac repair surgery for congenital heart disease with a mean age of 1-2 years to RIPC or conventional surgery. Troponin I levels and postoperative inotropic requirements were lower in the RIPC group as compared to the control group [21]. Several larger trials using RIPC in various clinical situations are ongoing (Table 1).

\section{Evaluation of Post-Conditioning in Humans}

Zhao et al. were the first to demonstrate that after 45 minutes of myocardial ischemia, coronary reperfusion coupled with three interspersed 30-second cycles of myocardial ischemia reduced infarct size in dogs from $47 \%$ to $11 \%$. They named this form of cardioprotection "ischemic postconditioning" [16]. Since the first animal study was published in 2003 several human studies have been conducted with positive results.

Our group showed in a pilot, non-randomized study that PosC was associated with significant reductions in infarct size and better cardiac function [22] (Table 2).

Staat et al. randomized 30 patients with acute ST-segment myocardial infarction (STEMI) to undergo primary angioplasty vs. primary angioplasty plus ischemic PosC. The authors found that PosC was associated with smaller infarct sizes (Figure 2), as determined by the area under the curve of CK release, better myocardial blush grades and more complete ST-segment resolution when compared to primary angioplasty alone [23]. The early benefits of PosC persisted over time, as evident by improved Ejection Fraction $(\mathrm{EF})$ in patients treated with PosC (56\%) relative to controls (49\%) ( $\mathrm{p}=0.04)$ [24].

Lønborg et al. evaluated the role of PosC in 118 patients undergoing primary angioplasty for the treatment of ST-elevation myocardial infarction. The primary end-point of the study was salvage index as assessed by cardiac Magnetic Resonance Imaging (MRI). The authors found a significant $31 \%$ increase in myocardial salvage index and a $19 \%$ reduction in infarct size among patients treated with PosC. Clinically, this benefit translated into less patients developing heart failure $(27 \%$ vs. $46 \%, \mathrm{p}=0.04)$ at follow-up [25].

Piot et al. used cyclosporine, an inhibitor of the mitochondrial transitional pore considered a postconditioning mimetic, to limit infarct size in patients undergoing primary PCI for STEMI. Opening of the mitochondrial transitional pore is believed to play a crucial role in reperfusion injury and $\mathrm{PosC}$, as well as $\mathrm{PreC}$, appear to block the opening of this pore (see next section). Cyclosporine, given at a dose of $2.5 \mathrm{mg}$ per kilogram of body weight, was associated with a significant reduction in infarct size assessed by CK release and cardiac MRI (Figure 3) [26].

\section{Potential Mechanisms of Pre-Conditioning and Post- conditioning}

Although reperfusion salvages myocardium that would ultimately die in its absence, restoring blood flow to the myocardium carries the potential to exacerbate reperfusion injury $[10,11]$. Reperfusion injury then offsets the optimal salvage of myocardium achieved during coronary angioplasty. Early strategies to attenuate reperfusion injury applied concepts derived from cardiac surgery, in which protecting the myocardium from ischemia-reperfusion injury was a mainstay of the operative strategy. Pre- and post-conditioning marshal a variety of endogenous mechanisms that operate at numerous levels and target a 
Citation: Iqbal Q, Garcia S (2013) Myocardial Protection through Pre- and Post-Conditioning: A Review of Mechanisms, Clinical Trials and Future Directions. J Cardiovasc Dis Diagn 1: 115. doi:10.4172/2329-9517.1000115

Page 3 of 5

\section{Title}

Effects of Remote Ischemic PreConditioning in Off-pump Versus On-pump

Coronary Artery Bypass Grafting (RIPCON)

Protective Effects of Long-term Remote Limb Ischemic Preconditioning For Carotid Artery Stenting

Clinical Trial on Remote Ischemic Preconditioning and Cerebral Small Vessel Disease (RIPC-SVD)

Remote Ischemic Preconditioning in Subarachnoid Hemorrhage (RIPC-SAH) NCT 01158508

Reduction of Myocardial Infarction by Preconditioning in Patients With Ruptured Abdominal Aortic Aneurysm (PreconRAAA)

Remote Ischemic Preconditioning in Neurological Death Organ Donors (RIPNOD)

Pilot Study of Remote Ischemic Preconditioning in Heart Failure

Remote Ischemic Preconditioning and Risk of Contrast-Induced Acute Kidney Injury in Patients Undergoing Coronary Stent Implantation (REPAIR)

Remote Ischemic Preconditioning Mechanism Study

Remote Ischaemic Preconditioning for Heart Surgery (RIPHeart-Study)

Limb Remote Ischemic Preconditioning for Heart and Intestinal Protection During Laparoscopic Colorectal Surgery

The Neuroprotection of Remote Ischemic Preconditioning (RIPC) on Cardiac Surgery in Multicenter

Remote Ischemic Preconditioning Prior to Vascular Surgery (CRIPES)

Remote Ischemic PreConditioning Effect on Postsurgical Pain (RIPCEPP)

Effect of Ketorolac and Remote Ischemic Preconditioning on Renal Ischemiareperfusion Injury in Patients Undergoing Partial Nephrectomy

Limb Ischemic Preconditioning for Prevention of Contrast Media Induced Nephropathy in Diabetic Kidney Patient

Remote Ischemic Preconditioning Combined to Local Ischemic Postconditioning in Acute MI (RIRE-1)

Seattle Cardiorenal Remote Ischemic Preconditioning Trial (SCRIPT)

Effect of Remote Ischemic Preconditioning on Incidence of Atrial Fibrillation in Patients Undergoing Coronary Artery Bypass Graft Surgery

Evaluation of a Local Preconditioning Effect in Patients Undergoing Cardiac Surgery

The Effect of Remote Ischemic Postconditioning on Liver Graft and Renal Function in Patients Undergoing Living-related Liver Transplantation

Study of Remote Ischemic Postconditioning

The Effect of Remote Postconditioning on Graft Function in Patients Undergoing Living-related Kidney Transplantation

Postconditioning in ST-elevation Myocardial Infarction (POSTEMI)

Phase II Clinical Trial to Evaluate the Benefits of Postconditioning in STElevation Myocardial Infarction (STEMI)

Ischemic Postconditioning in Cardiac Surgery (IPICS)

Delayed Postconditioning (PRIME)

Remote Ischemic Postconditioning During Percutaneous Coronary Interventions (RIP-PCI)

DANish Study of Optimal Acute Treatment of Patients With ST-elevation Myocardial Infarction (DANAMI-3)

Remote Ischemic Preconditioning Combined to Local Ischemic Postconditioning in Acute Myocardial Infarction (RIRE-1)

Effect of Remote Ischemic Preconditioning on Acute Kidney Injury in Adult Valve Replace

Effect of Remote Ischemic Preconditioning in Patient Undergoing Cardiac Bypass Surgery

Chronic Remote Ischemic Conditioning to Modify Post-MI Remodeling (CRIC$\mathrm{RCT}$ )
NCT 00883363

NCT 01515072

NCT 01067703

NCT 01128790

NCT 01827891

NCT 01541436

NCT 01832337

NCT 01231789

NCT 01558596

NCT 01383317

NCT 01836406

NCT 01763034

NCT 01390142

NCT 01260259

NCT 01500369

NCT 01482780

NCT 01637038

NCT 01450475

NCT 01363687

NCT 00922675

NCT 01324453

NCT 01813968

NCT 01483755

\section{Primary Outcome Measure}

Perioperative extent of myocardial injury

The Incidence of Brain or Cardiac Ischemic Events and Death

Changes in brain lesions of small vessel disease

Occurrence of neurological events, or vasospasm at day $3,7,15$ and 30

Reduction in myocardial infarction

Improvement in donor stability, organ quality, organ yield, and early post transplant clinical outcomes after preconditioning

Exercise performance - VO2, exercise time, exercise workload, anaerobic threshold

Acute kidney injury within $72 \mathrm{hr}$ after $\mathrm{PCl}$

Effect of RIPC on Areas of hyperalgesia and allodynia to mechanica stimuli.

Composite of all-cause mortality, non-fatal myocardial infarction, any new stroke, and/or acute renal failure

Protection of RIPC on heart and intestinal injury in patients undergoing elective laparoscopic colorectal surgery

Neurological injury markers

Troponin I elevation

Comparison of pain intensity and unpleasantness postoperatively

Serum creatinine at baseline, $2 \mathrm{hr}, 12 \mathrm{hr}, 24 \mathrm{hr}, 48 \mathrm{hr}, 72 \mathrm{hr}$ after clamping release

Kidney injury before and after coronary angiogram

Infarct size as assessed by $72 \mathrm{hrs}$ area under curve serum CK-MB \& cardiac magnetic resonance imaging at 3-month.

Incidence of acute kidney injury (AKI)

Post op atrial fibrillation

Enzyme leakage (Troponin T, CK-MB) will be measured preoperatively, at the beginning of the operation, the beginning of ECC at the time of reperfusion the arrival on the ICU and 6,12,24,48 and 72 hours postop and the area under the curve will be calculated

Total bilirubin before surgery and at $6,12,24,36,48,60,72,84,96$ hours after the end of surgery

The primary outcome is assessing whether cTnl or CK-MB concentration reduce at 24 hours after remote ischemic postconditioning

Renal function of recipient after living-related kidney transplantation Infarct size, assessed by MR

Myocardial Salvage Index

Change in cardiac index between the groups during the first postoperative day

Size of the infarct estimated by magnetic resonance imaging at day 5 post-reperfusion

To study the effects of different degrees of remote ischemic postconditioning on myocardial necrosis and inflammation following $\mathrm{PCl}$

Cardiac death, re-infarction, heart failure

NCT 01435408

NCT 01390142 Infarct size

NCT 01476969 Renal function

NCT 00397163 Troponin-T release over the perioperative 72-hour period

NCT 01817114 Change in LVEDV from baseline

Table 1: Ongoing clinical trials of myocardial pre-conditioning listed on clinicaltrials.gov website as of June 2013. 
broad range of pathological mechanisms [27-34]. These mechanisms include ligands, such as adenosine and opioids that act as proximal triggers to stimulate molecular pathways involving mediators such as protein kinase $\mathrm{C}$, mitochondrial ATP-sensitive potassium channels, and survival kinases [27-33]. Post-conditioning may also inhibit deleterious pathways such as p38 and JNK Mitogen-Activated Protein (MAP) kinases and attenuate the damage to endothelial cells and cardiomyocytes from oxidants, cytokines, proteases, and inflammatory cells [34-36]. Post-conditioning induces the expression of mRNA

\begin{tabular}{|c|c|c|c|c|c|}
\hline & N & Ischemic time (hs) & Age & CK (IU) & LVEF (\%) \\
\hline PostC & 22 & 4.5 & $61 \pm 3$ & $1987 \pm 281$ & $53 \pm 2$ \\
\hline Control & 21 & 4.5 & $56 \pm 3$ & $2335 \pm 434$ & $45 \pm 3$ \\
\hline
\end{tabular}

Table 2: Effect of PosC on infarct size and ventricular function in patients undergoing primary $\mathrm{PCl}$ for the treatment of acute myocardial infarction. Infarct size and ejection fraction were better among PosC treated patients. Adapted from Garcia $\mathrm{S}$ et al. [22] . PosC=Post-conditioning, $\mathrm{Ck}=$ Creatinine kinase, LVEF= Left Ventricular Ejection Fraction.

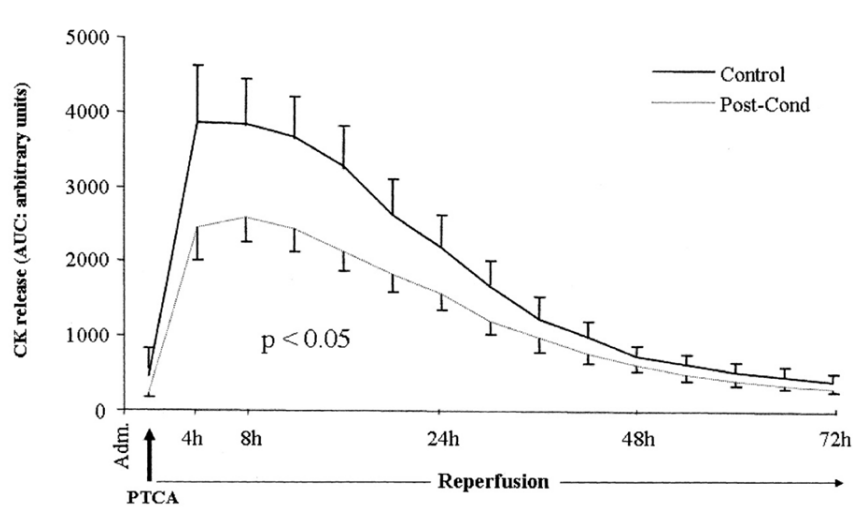

Figure 2: The area under the curve of $\mathrm{CK}$ release was significantly lower $(36 \%)$ in STEMI patients treated with Post- conditioning reproduced from Circulation Oct 4 2005; 112(14): 2143-2148 with permission of Wolters Kluwer Health.

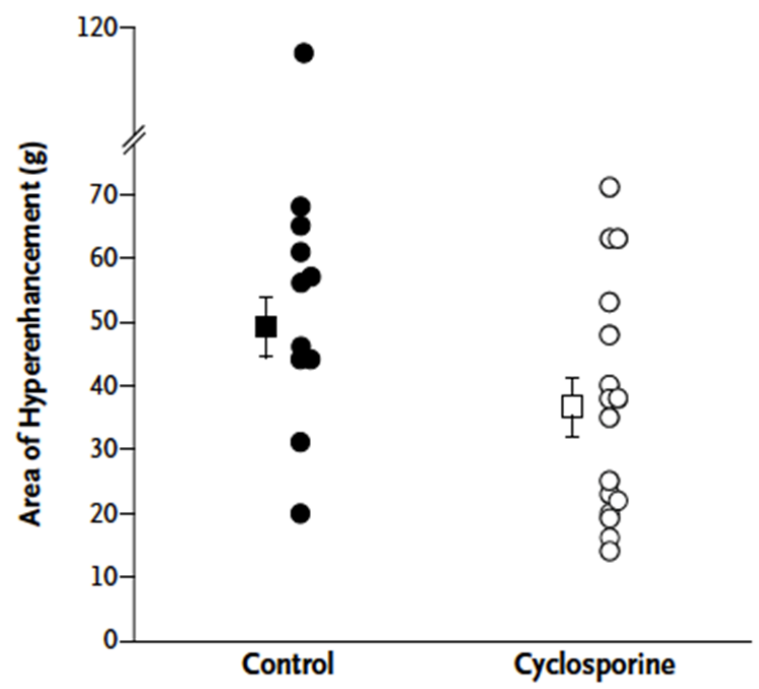

Figure 3: Infarct size, assessed by gadolinium enhancement, in patients treated with cyclosporine vs. Control. Cyclosporine was associated with a $20 \%$ reduction in infarct size. Reproduced from N Engl J Med. Jul 31 2008; 359(5): 473-481 with permission of Massachusetts Medical Society.

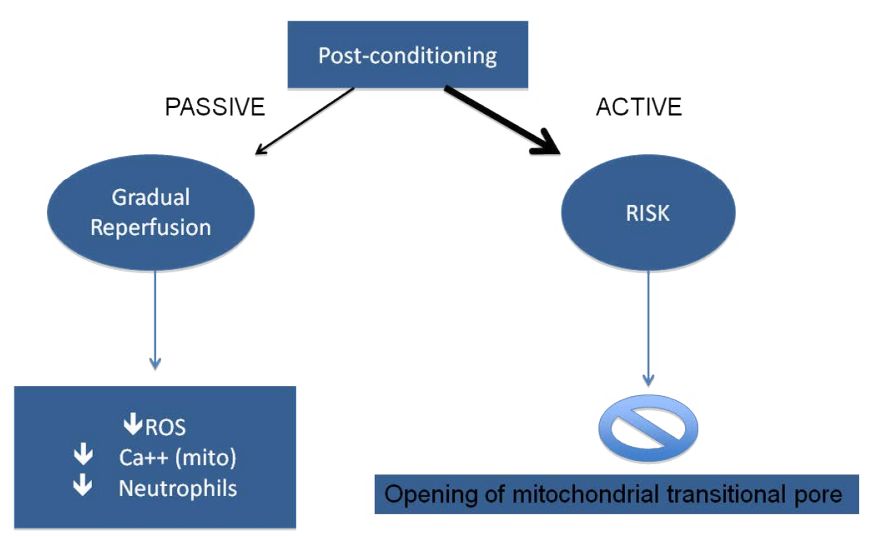

Figure 4: Potential Mechanisms of post- Conditioning. Reproduced from Circulation. 2005; 111: 194-197 with permission of Wolters Kluwer Health.

for iNOS; a key enzyme for the production of nitric oxide, which is a potent vasodilator [31-33]. At a cellular level, the effects of pre- and post-conditioning converge on the mitochondria, in particular the mitochondrial transition pore [37] (Figure 4). This pore opens during the first minutes of reperfusion in response to mitochondrial calcium overload and oxidative stress. Post-conditioning protects the heart through the inhibition of mitochondrial transition pore opening, which reduces calcium influx into the mitochondria [37]. Other salutary effects of PosC include less production of reactive oxygen species and inflammatory markers [34]. Post-conditioning is also thought to confer protection to the microcirculation [22,24,25]. Recent studies (including ours) have shown that PosC patients had increased TIMI frame counts and improved endothelium-dependent vasodilatation $[22,38]$.

\section{Future Directions}

Although recent advances in medical therapy, procedural techniques and integration of systems of care, cardiovascular disease continues to impose a heavy toll on society; both in terms of morbidity as well as financial costs. More recently the concept of protecting the myocardium through myocardial pre- and post-conditioning has evolved "from bench to bedside" with several small trials reporting positive results. As the field moves forward the next wave of clinical trials should focus on clinically meaningful end-points such as heart failure incidence, cardiovascular hospitalizations, arrhythmias, and cardiovascular death. To have enough power and external validity these trials should involve multiple centers, which will not be easy without industry or government support. Mechanistic studies are also needed to better understand the underpinnings of cardiac protection and provide a framework in which new pharmacological agents can be developed.

\section{Acknowledgements}

Dr. Garcia is a recipient of a career development award (1IK2CX000699-01) from the VA Office of Research and Development.

\section{References}

1. Roger VL, Go AS, Lloyd-Jones DM, Benjamin EJ, Berry JD, et al. (2012) Executive summary: heart disease and stroke statistics--2012 update: a report from the American Heart Association. Circulation. 125: 188-197.

2. Anderson JL, Marshall HW, Bray BE, Lutz JR, Frederick PR, et al. (1983) A randomized trial of intracoronary streptokinase in the treatment of acute myocardial infarction. N Engl J Med 308: 1312-1318.

3. Keeley EC, Boura JA, Grines CL (2003) Primary angioplasty versus intravenous 
Citation: Iqbal Q, Garcia S (2013) Myocardial Protection through Pre- and Post-Conditioning: A Review of Mechanisms, Clinical Trials and Future Directions. J Cardiovasc Dis Diagn 1: 115. doi:10.4172/2329-9517.1000115

Page 5 of 5

thrombolytic therapy for acute myocardial infarction: a quantitative review of 23 randomised trials. Lancet 361: 13-20.

4. Weaver WD, Simes RJ, Betriu A, Grines CL, Zijlstra F, et al. (1997) Comparison of primary coronary angioplasty and intravenous thrombolytic therapy for acute myocardial infarction: a quantitative review. JAMA 278: 2093-2098.

5. Ting HH, Rihal CS, Gersh BJ, Haro LH, Bjerke CM, et al. (2007) Regional systems of care to optimize timeliness of reperfusion therapy for ST-elevation myocardial infarction: the Mayo Clinic STEMI Protocol. Circulation 116: 729736

6. Lloyd-Jones DM, Larson MG, Leip EP, Beiser A, D'Agostino RB, et al. (2002) Lifetime risk for developing congestive heart failure: the Framingham Heart Study. Circulation 106: 3068-3072.

7. Bristow MR, Saxon LA, Boehmer J, Krueger S, Kass DA, et al. (2004) Cardiacresynchronization therapy with or without an implantable defibrillator in advanced chronic heart failure. N Engl J Med 350: 2140-2150.

8. Bardy GH, Lee KL, Mark DB, Poole JE, Packer DL, et al. (2005) Amiodarone or an implantable cardioverter-defibrillator for congestive heart failure. $\mathrm{N}$ Eng J Med 352: 225-237.

9. Bolli R, Becker L, Gross G, Mentzer R Jr, Balshaw D, et al. (2004) Myocardial protection at a crossroads: the need for translation into clinical therapy. Circ Res 95: 125-134.

10. Yellon DM, Hausenloy DJ (2007) Myocardial reperfusion injury. N Engl J Med 357: 1121-1135

11. Braunwald E, Kloner RA (1985) Myocardial reperfusion: a double-edged sword? J Clin Invest 76: 1713-1719.

12. Yellon DM, Baxter GF (1999) Reperfusion injury revisited: is there a role for growth factor signaling in limiting lethal reperfusion injury? Trends Cardiovasc Med 9: 245-249.

13. Tsao PS, Aoki N, Lefer DJ, Johnson G 3rd, Lefer AM (1990) Time course of endothelial dysfunction and myocardial injury during myocardial ischemia and reperfusion in the cat. Circulation 82: 1402-1412.

14. Roubille F, Franck-Miclo A, Covinhes A, Lafont C, Cransac F, et al. (2011) Delayed postconditioning in the mouse heart in vivo. Circulation 124: 13301336.

15. Murry CE, Jennings RB, Reimer KA (1986) Preconditioning with ischemia: a delay of lethal cell injury in ischemic myocardium. Circulation 74: 1124-1136.

16. Zhao ZQ, Corvera JS, Halkos ME, Kerendi F, Wang NP, et al. (2003) Inhibition of myocardial injury by ischemic postconditioning during reperfusion: comparison with ischemic preconditioning. Am J Physiol Heart Circ Physiol 285: H579-588.

17. Przyklenk K, Bauer B, Ovize M, Kloner RA, Whittaker P (1993) Regional ischemic 'preconditioning' protects remote virgin myocardium from subsequent sustained coronary occlusion. Circulation 87: 893-899.

18. Hoole SP, Heck PM, Sharples L, Khan SN, Duehmke R, et al. (2009) Cardiac Remote Ischemic Preconditioning in Coronary Stenting (CRISP Stent) Study: a prospective, randomized control trial. Circulation 119: 820-827.

19. Hausenloy DJ, Mwamure PK, Venugopal V, Harris J, Barnard M, et al. (2007) Effect of remote ischaemic preconditioning on myocardial injury in patients undergoing coronary artery bypass graft surgery: a randomised controlled trial. Lancet 370: $575-579$

20. Ali ZA, Callaghan CJ, Lim E, Ali AA, Nouraei SA, et al. (2007) Remote ischemic preconditioning reduces myocardial and renal injury after elective abdominal aortic aneurysm repair: a randomized controlled trial. Circulation 116: 198-105.

21. Cheung MM, Kharbanda RK, Konstantinov IE, Shimizu M, Frndova H, et al. (2006) Randomized controlled trial of the effects of remote ischemic preconditioning on children undergoing cardiac surgery: first clinical application in humans. J Am Coll Cardiol 47: 2277-2282.

22. Garcia S, Henry TD, Wang YL, Chavez IJ, Pedersen WR, et al. (2011) Long term follow-up of patients undergoing postconditioning during ST-elevation myocardial infarction. J Cardiovasc Transl Res 4: 92-98.

23. Staat $P$, Rioufol G, Piot C, Cottin $Y$, Cung TT, et al. (2005) Postconditioning the human heart. Circulation 112: 2143-2148.

24. Thibault H, Piot C, Staat P, Bontemps L, Sportouch C, et al. (2008) Long-term benefit of postconditioning. Circulation 117: 1037-1044.

25. Lønborg J, Kelbaek H, Vejlstrup N, Jørgensen E, Helqvist S, et al. (2010) Cardioprotective effects of ischemic postconditioning in patients treated with primary percutaneous coronary intervention, evaluated by magnetic resonance. Circ Cardiovasc Interv 3: 34-41.

26. Piot C, Croisille P, Staat P, Thibault H, Rioufol G, et al. (2008) Effect of cyclosporine on reperfusion injury in acute myocardial infarction. N Engl J Med 359: 473-481.

27. Fryer RM, Auchampach JA, Gross GJ (2002) Therapeutic receptor targets of ischemic preconditioning. Cardiovasc Res 55: 520-525

28. Olafsson B, Forman MB, Puett DW, Pou A, Cates CU, et al. (1987) Reduction of reperfusion injury in the canine preparation by intracoronary adenosine: importance of the endothelium and the no-reflow phenomenon. Circulation 76 1135-1145.

29. Gross ER, Hsu AK, Gross GJ (2004) Opioid-induced cardioprotection occurs via glycogen synthase kinase beta inhibition during reperfusion in intact rat hearts. Circ Res 94: 960-966.

30. Patel HH, Moore J, Hsu AK, Gross GJ (2002) Cardioprotection at a distance: mesenteric artery occlusion protects the myocardium via an opioid sensitive mechanism. J Mol Cell Cardiol 34: 1317-1323.

31. Sato T, O'Rourke B, Marbán E (1998) Modulation of mitochondrial ATP dependent $\mathrm{K}+$ channels by protein kinase C. Circ Res 83: 110-114.

32. Ytrehus K, Liu Y, Downey JM (1994) Preconditioning protects ischemic rabbit heart by protein kinase C activation. Am J Physiol 266: H1145-1152.

33. Konstantinov IE, Li J, Cheung MM, Shimizu M, Stokoe J, et al. (2005) Remote ischemic preconditioning of the recipient reduces myocardial ischemiareperfusion injury of the denervated donor heart via a Katp channel-dependent mechanism. Transplantation. 79: 1691-1695.

34. Vinten-Johansen J, Jiang R, Reeves JG, Mykytenko J, Deneve J, et al. (2007) Inflammation, proinflammatory mediators and myocardial ischemia-reperfusion Injury. Hematol Oncol Clin North Am 21: 123-145.

35. Vinten-Johansen J (2007) Postconditioning: a mechanical maneuver that triggers biological and molecular cardioprotective responses to reperfusion. Heart Fail Rev 12: 235-244.

36. Vinten-Johansen J, Zhao ZQ, Jiang R, Zatta AJ, Dobson GP (2007) Preconditioning and postconditioning: innate cardioprotection from ischemiareperfusion injury. J Appl Physiol 103: 1441-1448.

37. Argaud L, Gateau-Roesch O, Raisky O, Loufouat J, Robert D, et al. (2005) Postconditioning inhibits mitochondrial permeability transition. Circulation 111 : 194-197.

38. Ma XJ, Zhang XH, Li CM, Luo M (2006) Effect of postconditioning on coronary blood flow velocity and endothelial function in patients with acute myocardial infarction. Scand Cardiovasc J 40: 327-333. 